Juan Garrido, Francisco Vázquez, Fernando Morilla, Inverted decoupling internal model control for square stable multivariable time delay systems, Journal of Process Control, Volume 24, Issue 11, November 2014, Pages 1710-1719, ISSN 0959-1524, http://dx.doi.org/10.1016/j.jprocont.2014.09.003. (http://www.sciencedirect.com/science/article/pii/S0959152414002339)

\title{
INVERTED DECOUPLING INTERNAL MODEL CONTROL FOR SQUARE STABLE MULTIVARIABLE TIME DELAY SYSTEMS
}

\author{
Juan Garrido $^{\mathrm{a}^{*}}$, Francisco Vázquez ${ }^{\mathrm{a}}$, Fernando Morilla ${ }^{\mathrm{b}}$ \\ ${ }^{a}$ Department of Computer Science and Numerical Analysis, University of Cordoba, \\ Campus de Rabanales, 14071, Cordoba, Spain \\ *Fax: (+34)957218729; e-mail: juan.garrido@uco.es \\ ${ }^{b}$ Department of Computer Science and Automatic Control, UNED, Juan del Rosal 16, \\ 28040, Madrid, Spain
}

\begin{abstract}
This paper presents a new tuning methodology of the main controller of an internal model control structure for $n \times n$ stable multivariable processes with multiple time delays based on the centralized inverted decoupling structure. Independently of the system size, very simple general expressions for the controller elements are obtained. The realizability conditions are provided and the specification of the closed-loop requirements is explained. A diagonal filter is added to the proposed control structure in order to improve the disturbance rejection without modifying the nominal set-point response. The effectiveness of the method is illustrated through different simulation examples in comparison with other works.
\end{abstract}

Keywords: internal model control; multivariable time delay systems; decoupling control.

\section{Introduction}

Time delays arise in many industrial processes as a consequence of different phenomena such as transport times of mass, information or energy; accumulation of time lags in processes interconnected in series; or processing time [1]. Time delays affect the performance of traditional control systems because they can lead to very poor system response as they prevent high controller gain from be used in order to avoid instability. The Smith Predictor (SP) was the first compensator specially designed for single-input single output (SISO) systems with time delay [2]. It allows the elimination of the time delay in the characteristic equation. In the last years, different modifications of the SP have been developed to overcome some drawbacks of its initial proposal and to improve its performance $[3,4]$. 
In multiple-inputs multiple-outputs (MIMO) systems there may be important couplings between inputs and outputs signals which may complicate the feedback controller design. In presence of time delays this design becomes even more difficult because each output is affected by each input with different time delays [5]. As a result, a transfer function matrix representation of the MIMO process is preferred in these cases[6]. Different approaches have been developed in order to design controller for multivariable systems with multiple time delays: some authors have extended the SP to the multivariable case [7-9]. In line with the previous ones, other works propose to solve the problem by means of an internal model control (IMC) applied to MIMO processes [10,11]. There is a close relationship between PS and IMC [12] since the SP can expressed in an equivalent IMC structure as shown in Figure 1 where $G_{m}$ is the multi-delay model of the plant. Others authors develop directly multivariable methodologies based on the conventional unity feedback structure: decoupling control [13-16],multivariable PID controllers [17, 18], $\mathrm{H}_{\infty}$ controllers [19], or decentralized controllers [20, 21]. Some of these methodologies combine PS or IMC with some of the last methods [6, 22]. Others use two degree of freedom control structures in order to achieve a good performance for reference tracking and disturbance rejection separately $[23,24]$.

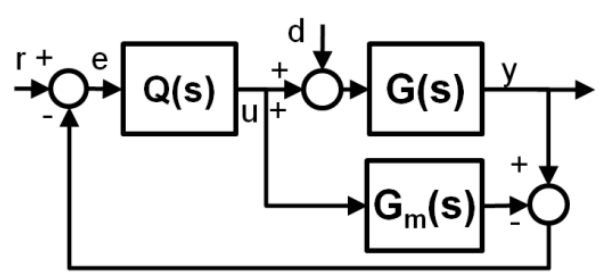

Figure 1. IMC scheme.

In order to apply IMC to multivariable systems, two approaches can be usually found.The first one consists in designing a decoupler $D(s)$ to the original process in order to obtain a diagonal or diagonal dominant apparent process, and then, applying the IMC to this apparent process G(s) $D(s)$ (Figure 2) [25, 26]. The IMC design can be performed as that of SISO case. The secondone and more commonapplies simultaneously decoupling control and IMC $[6,27]$ using the scheme of Figure 1.

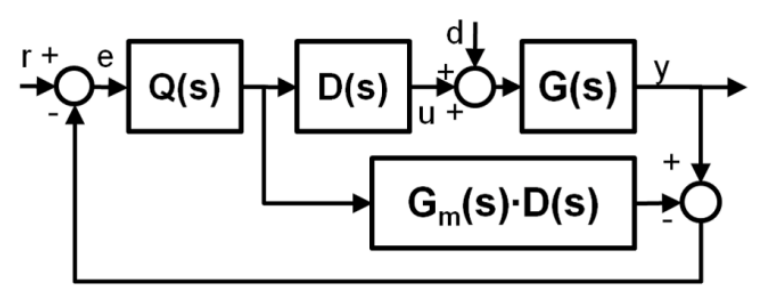

Figure 2. IMC with decoupler scheme.

From the IMC scheme in Figure 1, the matrix expressions of the closed-loop transfer matrix T(s) from the references $r$ to the outputs $y$, and the transfer matrix $\mathrm{H}(\mathrm{s})$ from the load disturbances $d$ to the outputs $y$ can be obtained as follows: 


$$
\begin{aligned}
& T(s)=G(s) \cdot Q(s) \cdot\left[I+\left(G(s)-G_{m}(s)\right) \cdot Q(s)\right]^{-1} \\
& H(s)=G(s)-G(s) \cdot\left[I+Q(s)\left(G(s)-G_{m}(s)\right)\right]^{-1} \cdot Q(s) \cdot G(s)
\end{aligned}
$$

where $G(s), G_{m}(s)$ and $Q(s)$ are the transfer matrix of the plant, the nominal model of the plant and the main controller of the IMC structure, respectively. When the model of the process is perfect, i.e. $G_{m}(s)=G(s)$, the previous closed-loop transfer matrixes are simplified to (3) and (4).

$$
\begin{aligned}
& T(s)=G(s) \cdot Q(s) \\
& H(s)=G(s)-G(s) \cdot Q(s) \cdot G(s)=(I-T(s)) \cdot G(s)
\end{aligned}
$$

Therefore, the main controller Q(s) can be calculated from (3) after defining the closedloop transfer matrix T(s) properly for realizability and stability. Most of multivariable IMC methodologies use a transfer matrix $\mathrm{Q}(\mathrm{s})$ in which the process inputs $u$ are derived by a time-weighted combination of the error signals $e$. If decoupling is required in T(s), the main problem of such methods is the increase of the design complication when the size of the system is large, because the calculations become more complex and important approximations are usually required. For instance, an analytical decoupling IMC method is developed in [11] on the basis of the $\mathrm{H}_{2}$ optimal performance objective. It uses the IMC scheme of Figure 1 and complex controller elements are obtained for the ideal optimal control matrix even for $2 \times 2$ processes.

This work proposes a new tuning methodology of the main controller of an IMC structure for directly decoupling and stabilizing square stable multivariable processes with multiple time delays. It is based on the structure of centralized inverted decoupling [28] that allows obtaining very simple expressions for controller elements independently of the system size. However, as disadvantage, it cannot be applied to processes with multivariable zeros in the right half plane (RHP) since it results unstable. The paper is structured as follows. In Section 2, the proposed method is developed for $n \times n$ processes. Several aspects as realizability are discussed. The equivalency between multivariable IMC and centralized inverted decoupling control schemes is shown. In order to improve disturbance rejection a diagonal filter in the feedback loop is proposed. Section 3 illustrates the methodology with several simulation examples. Finally, conclusions are summarized in Section 4.

\section{Inverted decoupling IMC}

\subsection{General expressions for $n \times n$ processes}

Assuming a stable square process G(s) with $n$ inputs and $n$ outputs, the proposed methodology uses the centralized inverted decoupling control to design the control matrix Q(s) obtaining a decoupled response in T(s). As shown in Figure 3, Q(s) is split 
into two blocks: a matrix Qd(s) in the direct path (between the error signalse and the control signals $u$ ) and a matrix Qo(s) in a feedback loop (in the opposite direction). According to the inverted decoupling structure, Qd(s) must have only $n$ elements different from zero which connect the error signals $e$ with the control signals $u$. In order to decouple the system, Qo(s) feeds back the control signals $u$ toward the controller inputs. Qo(s) must have only $n$ zero elements, which correspond with the transpose nonzero elements of Qd(s). For example, in a $4 \times 4$ process, if element Qd(1,4) is selected as a direct connection between $u_{1}$ and $e_{4}$, there will not be feedback from $u_{1}$ to $e_{4}$ and consequently, Qo $(4,1)$ must be zero.

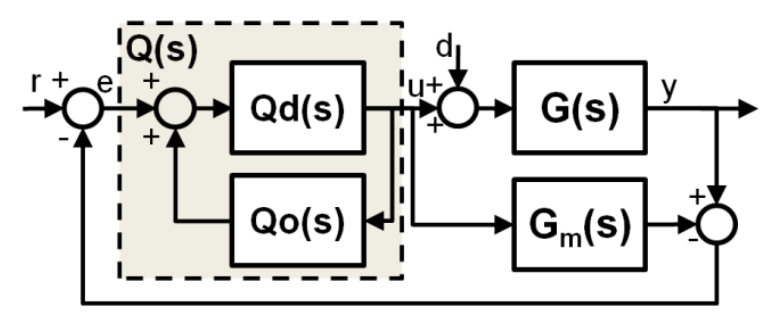

Figure 3. Inverted decoupling IMC scheme.

From the representation in Figure 3 and from the general IMC equation (3), the expression of the elements of Qd(s) and Qo(s) can be calculated by means of (5).

$Q d^{-1}(s)-Q o(s)=T^{-1}(s) \cdot G(s)$

This main expression is quite similar to that obtained for the centralized inverted decoupling control in [28]. However, in this method, the desired open-loop transfer matrix L(s) used in [28] is replaced by the desired closed-loop transfer matrix T(s) because of the IMC structure. Afterward, the design equations are practically the same of [28]. Nevertheless, the tuning procedure using T(s) instead of L(s) allows specifyingthe desired nominal performance in a more direct and easier way than that used in [28]. In the last one, using the classical control feedback scheme,specifications need to be translated to the open-loop transfer functions from the closed-loop requirements.

Assuming that the desired closed-loop response is a decoupled response from the references to the outputs, the matrix $\mathrm{T}(\mathrm{s})$ must be diagonal. Then, the main advantage of (5) over expressions of conventional multivariable IMC methods is its simplicity, regardless of the size of the process, because the resulting subtraction of $\mathrm{Qd}^{-1}(\mathrm{~s})$ and $\mathrm{Qo}(\mathrm{s})$ is a transfer matrix with only one element to be calculated for each position.

Note that Qd(s) has to be non-singular since it is inverted, and therefore, when its nonzero elements are chosen, only one element in each row and column can be selected. As a result, for an $n \times n$ system there are $n$ ! possible configurations of Qd(s). To name them, the authors propose the same notation in [29], in which the indicated number corresponds to the column with the chosen element. For instance, in a $2 \times 2$ system there 
are two configurations: 1-2 when elements $\mathrm{Qd}(1,1)$ and $\mathrm{Qd}(2,2)$ are selected to be nonzero; 2-1 when elements $\mathrm{Qd}(1,2)$ and $\mathrm{Qd}(2,1)$ are chosen. The expression of the controller elements for each configuration is different, which is interesting because some choices can result in non-realizable elements. Therefore, the configuration can be selected depending on the realizability, which will be discussed later.

The general expressions for $2 \times 2$ processes can be derived from (5) easily. For instance, assuming configuration 1-2, the following matrix equation is obtained:

$$
\begin{aligned}
& \left(\begin{array}{cc}
q d_{11}(s) & 0 \\
0 & q d_{22}(s)
\end{array}\right)^{-1}-\left(\begin{array}{cc}
0 & q o_{12}(s) \\
q o_{21}(s) & 0
\end{array}\right)= \\
= & \left(\begin{array}{cc}
\frac{1}{q d_{11}(s)} & -q o_{12}(s) \\
-q o_{21}(s) & \frac{1}{q d_{22}(s)}
\end{array}\right)=\left(\begin{array}{cc}
\frac{g_{11}(s)}{t_{1}(s)} & \frac{g_{12}(s)}{t_{1}(s)} \\
\frac{g_{21}(s)}{t_{2}(s)} & \frac{g_{22}(s)}{t_{2}(s)}
\end{array}\right)
\end{aligned}
$$

Then, the general expressions for the non-zero controller elements selecting configuration 1-2 are given by (7).

$$
\begin{aligned}
& q d_{11}(s)=\frac{t_{1}(s)}{g_{11}(s)} \quad q o_{12}(s)=\frac{-g_{12}(s)}{t_{1}(s)} \\
& q o_{21}(s)=\frac{-g_{21}(s)}{t_{2}(s)} \quad q d_{22}(s)=\frac{t_{2}(s)}{g_{22}(s)}
\end{aligned}
$$

In the same way, assuming configuration 2-1, the matrix equation in (8) is achieved and from it, the general expressions can be easily derived.

$$
\left(\begin{array}{cc}
-q o_{11}(s) & \frac{1}{q d_{21}(s)} \\
\frac{1}{q d_{12}(s)} & -q o_{22}(s)
\end{array}\right)=\left(\begin{array}{ll}
\frac{g_{11}(s)}{t_{1}(s)} & \frac{g_{12}(s)}{t_{1}(s)} \\
\frac{g_{21}(s)}{t_{2}(s)} & \frac{g_{22}(s)}{t_{2}(s)}
\end{array}\right)
$$

For higher dimensional system, the procedure is the same: to obtain the expressions from (5) after choosing the configuration. For $n \times n$ processes, if the configuration $p_{1}-p_{2}-$ $\ldots-p_{i}-\ldots-p_{n-1}-p_{n}$ is chosen, the non-zero elements of the $\mathrm{Qd}(\mathrm{s})$ and $\mathrm{Qo}(\mathrm{s})$ matrices are provided in (9) and (10), respectively. The transfer functions of the desired closed-loop processes $t_{i}(\mathrm{~s})$ can be specified in any way that assures the realizability of these controller elements.

$$
\begin{aligned}
& q d_{i j}(s)=\frac{t_{j}(s)}{g_{j i}(s)} \quad \forall i ; \quad j=p_{i} \\
& q o_{i j}(s)=\frac{-g_{i j}(s)}{t_{i}(s)} \quad \forall i, j ; / i \neq p_{j}
\end{aligned}
$$


From these general expressions, it can be concluded that the complexity of the controller elements in Qd(s) and Qo(s) is always the same, independent of the system size. With conventional schemes in Q(s), these elements become more complex as the size of the process increases.However, it is necessary to indicate that the proposed methodologyhas the same disadvantage as inverted decoupling: it cannot be applied to processes with multivariable RHP zeros, that is, RHP zeros in its determinant [28, 29]. If a RHP zero is specified in somet $t_{i}(\mathrm{~s})$ transfer function, it will appear as unstable pole in some $q o_{i j}(\mathrm{~s})$ elements. The case of RHP zeros associated to a single output is an exception. In this case, although the RHP zero is specified in the corresponding closedloop transfer function $t_{i}(\mathrm{~s})$, it will be canceled in the controller elements [29].

\subsection{Realizability and closed-loopperformance}

The realizability requirement for $\mathrm{Qd}(\mathrm{s})$ and $\mathrm{Qo}(\mathrm{s})$ is that all of their elements must be stable, causal and proper. For processes with time delays or RHP zeros, direct calculations can lead to elements with prediction or unstable poles. In the proposed methodology, there are two issues regarding controller realizability that have to be analyzed: firstly, it is necessary to check if it is possible to achieve realizability using the selected configuration; and secondly, after confirming the previous condition, it is essential to determine how to specify the desired closed-loop transfer functions $t_{i}(\mathrm{~s})$. Next, the conditions that a specified configuration needs to fulfill to be realizable are provided. In addition, the constraints on the closed-loop processes to achieve such realizability are indicated as well.

In the controller expressions (9) and (10), each desired closed-loop transfer function $t_{i}$ (s) appears associated to the process transfer functions $g_{i j}(\mathrm{~s})$ of the same row $i$. Thus, there are three aspects to take into consideration and to be inspected for each row of the plantmatrix $\mathrm{G}(\mathrm{s})$ in order to avoid non-causal time delays, negative relative degrees and RHP zeros becoming unstable poles.

This study can be carried out similarly to that in [28]. If $g_{i k}(\mathrm{~s})$ is the transfer function of the row $i$ with the smallest time delay $\theta_{i k}$, the smallest relative degree $r_{i k}$, or the smallest RHP zero multiplicity $\eta_{i k}$ of each RHP zero of the row, the element $q d_{k i}(\mathrm{~s})$ of $\mathrm{Qd}(\mathrm{s})$ should be chosen to be in the direct path between the error signals and the control signals (it should be non-zero). This transfer function $g_{i k}(\mathrm{~s})$ will appear in the denominator of the corresponding controller element $q d_{k i}(\mathrm{~s})$ according to (9). The numerator of this controller element is given by the desired closed-loop transfer function $t_{i}(\mathrm{~s})$ which must be specified with a time delay, relative degree, and RHP zero multiplicity equal or greater than that of $g_{i k}(\mathrm{~s})$ to achieve realizability in $q d_{k i}(\mathrm{~s})$. In contrast, according to (10), $t_{i}(\mathrm{~s})$ appears in the denominator of the corresponding controller elements $q o_{i j}(\mathrm{~s})$. Therefore, for realizability, the three previous aspects of $t_{i}(\mathrm{~s})$ must be specified less or equal than the least ones of the other $g_{i j}(\mathrm{~s})$ elements different from $g_{i k}(\mathrm{~s})$ in the row $i$. These conditions over time delay $\theta_{i}$, relative degree $r_{i}$ and RHP 
zero multiplicity $\eta_{i}$ of the closed-loop transfer function $t_{i}$ (s) can be expressed in the following constraints:

$$
\begin{aligned}
& \theta_{i k} \leq \theta_{i} \leq \min _{j \neq k}\left(\theta_{i j}\right), \\
& r_{i k} \leq r_{i} \leq \min _{j \neq k}\left(r_{i j}\right), \\
& \eta_{i k} \leq \eta_{i} \leq \min _{j \neq k}\left(\eta_{i j}\right) .
\end{aligned}
$$

The last condition must be fulfilled for each different multivariable RHP zero $z_{x}$ of the row $i$.

For a given configuration, conditions (11), (12) and (13) must be fulfilled for realizability when $t_{i}(\mathrm{~s})$ is specified. Nevertheless, for best performance of the control system it is undesirable to include any RHP zero or time delay in $t_{i}(\mathrm{~s})$ more than necessary. Therefore, $t_{i}(\mathrm{~s})$ is defined with the minimum time delay and minimum RHP zero multiplicity which fulfill the realizability conditions (11) and (13), that is, the minimum values by row $i\left(\theta_{i}=\theta_{i k}\right.$ and $\left.\eta_{i}=\eta_{i k}\right)$. The following form of $t_{i}(\mathrm{~s})$ is suggested:

$$
t_{i}(\mathrm{~s})=e^{-\theta_{i} s} \cdot \prod_{x=1}^{N z}\left(\frac{-s+z_{x}}{s+z_{x}}\right)^{\eta x_{i}} \cdot \frac{1}{\left(\lambda_{i} s+1\right)^{r_{i}}}
$$

wherethe time constant $\lambda_{i}$ determines the bandwidth of the closed-loopi and acts as a tuning parameter for performance and robustness, $\mathrm{Nz}$ is the total number of individual RHP zeros of the row $i$, and $\eta x_{i}$ is the proper multiplicity of the zero $z_{x}$ according to condition (13). The multiplicity $r_{i}$ of the associated pole is specified to provide the necessary high frequency roll-off rate being limited by the realizability condition (12). If relative degree $r_{i}$ must be specified zero in (14) according to this condition (12), there would be no tuning parameter. In this case, expression (15) is proposed instead of (14). It has an extra zero that can be used as an extra tuning parameter.

$$
t_{i}(\mathrm{~s})=e^{-\theta_{i} s} \cdot \prod_{x=1}^{N z}\left(\frac{-s+z_{x}}{s+z_{x}}\right)^{n x_{i}} \cdot \frac{\left(\gamma_{i} s+1\right)}{\left(\lambda_{i} s+1\right)}
$$

Assuming a realizable configuration in a $n \times n$ system with all of the elements of the row ias first order plus time delay (FOPTD) processes, the previous realizabilityconditions point out that the closed-loop transfer function $t_{i}(\mathrm{~s})$ has a relative degree equal to the unity, no RHP zeros and a time delay given by the minimum time delay of the associated row $i$. Therefore, $t_{i}(\mathrm{~s})$ is defined as follows:

$$
t_{i}(s)=\frac{e^{-s \theta i}}{\lambda_{i} s+1}
$$

where $\lambda_{\mathrm{i}}$ is the desired closed-loop time constant for reference tracking. 
When two or more elements of $\mathrm{Qd}(\mathrm{s})$ must be selected necessarily in the same column to fulfill the realizabilityconditions in all rows, there are no realizable configurations. Then, it is required to insert an additional diagonal block N(s) between the process $\mathrm{G}(\mathrm{s})$ and the controller in order to modify the system trying to force the non-realizable elements into realizability. Then, the methodology is applied to the new process $\mathrm{G}^{\mathrm{N}}(\mathrm{s})=\mathrm{G}(\mathrm{s}) \cdot \mathrm{N}(\mathrm{s})$.

$\mathrm{N}(\mathrm{s})$ is a diagonal transfer matrix with the necessary extra dynamics. If there are no realizability problems in the row $i$, the $n_{i i}(\mathrm{~s})$ element is equal to the unity. Otherwise, the required extra dynamics (time delay, pole or RHP zero) is added with the proper multiplicity to fulfill the corresponding realizability condition. In general, $n_{i i}(\mathrm{~s})$ is defined according to (17). Generally, it is preferable to add the minimum extra dynamics. Therefore, after checking the required additional dynamics of each configuration, it is selected that one with fewer RHP zeros or time delays in N(s). More detailed information about this issue is provided in [29].

$n_{i i}(\mathrm{~s})=e^{-\theta_{i i} s} \cdot \frac{1}{(\lambda s+1)^{r_{i i}}} \cdot \prod_{x=1}^{N z}\left(\frac{-s+z_{x}}{s+z_{x}}\right)^{\eta x_{i i}}$

For illustration, the following example is considered:

$G(s)=\left(\begin{array}{cc}\frac{(-s+0.2)^{2} \cdot e^{-9 s}}{(s+3)^{3}} & \frac{(-s+0.2) \cdot e^{-3 s}}{(s+3)^{2}} \\ \frac{e^{-7 s}}{s+3} & \frac{-e^{-2 s}}{s+3}\end{array}\right)$

This system has a multivariable RHP zero at $s=0.2$. Nevertheless, it is associated with a single output, the first one, and therefore, the proposed method can be applied. This RHP zero appears in the two process transfer functions of the first row with different multiplicity. According to the previous RHP zero condition, the element $q d_{12}$ (s) must be chosen to be non-zero in the Qd(s) because element $g_{12}(\mathrm{~s})$ has the smallest RHP zero multiplicity. In addition, it has the smallest time delay of the first row. In the second row, due to time delay condition, the element $q_{22}$ (s) should be selected to be non-zero in the Qd(s). Nevertheless, no configuration is initially realizable because elements $q d_{12}(\mathrm{~s})$ and $q d_{22}(\mathrm{~s})$ are in the same column. To attain realizability, an extra time delay of 5 units needs to be added in the second input $\left(n_{22}(s)=e^{-5 s}\right)$. In this case, the new process is given by (19), and using configuration 2-1, the element $q d_{11}$ (s) can be selected in the second row.

$$
G^{N}(s)=\left(\begin{array}{cc}
\frac{(-s+0.2)^{2} \cdot e^{-9 s}}{(s+3)^{3}} & \frac{(-s+0.2) \cdot e^{-8 s}}{(s+3)^{2}} \\
\frac{e^{-7 s}}{s+3} & \frac{-e^{-7 s}}{s+3}
\end{array}\right)
$$


Then, according to constraints (11), (12), (13) and (14), the closed-loop transfer functions can be defined by (20), assuming $\lambda_{1}=\lambda_{2}=1$. The RHP zero appears in the first closed-loop process, which is necessary to obtain stable controller elements.

$t_{1}(\mathrm{~s})=\frac{e^{-8 s}(-s+0.2)}{(s+0.2)(s+1)} \quad t_{2}(\mathrm{~s})=\frac{e^{-7 s}}{(s+1)}$

Finally, using the expressions in (8) the following controller matrices Qd(s) and Qo(s) are achieved:

$$
\begin{aligned}
& \operatorname{Qd}(s)=\left(\begin{array}{cc}
0 & \frac{(s+3)}{(s+1)} \\
\frac{(s+3)^{2}}{(s+1)(s+0.2)} & 0
\end{array}\right) \\
& \operatorname{Qo}(s)=\left(\begin{array}{cc}
\frac{-(-s+0.2)(s+0.2)(s+1) \cdot e^{-s}}{(s+3)^{3}} & 0 \\
0 & \frac{s+1}{s+3}
\end{array}\right)
\end{aligned}
$$

\subsection{Equivalent centralized controller}

The proposed inverted decoupling IMC scheme,depicted in Figure 3, can be transformed into a centralized inverted decoupling scheme (Figure 4). This can be interesting for an alternative implementation of the same controller. To do so, the nominal process model $\mathrm{G}_{\mathrm{m}}(\mathrm{s})$ and the controller matrix Qo(s) are combined into an only matrix $\mathrm{Ko}(\mathrm{s})=\mathrm{Qo}(\mathrm{s})+\mathrm{G}_{\mathrm{m}}(\mathrm{s})$. The matrix $\mathrm{Kd}(\mathrm{s})$ would be equal to $\mathrm{Qd}(\mathrm{s})$.

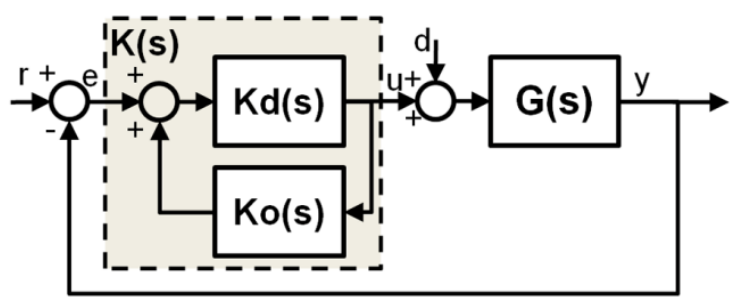

Figure 4. Centralized inverted decoupling control scheme.

For a particular configuration $p_{1}-p_{2}-\ldots-p_{i}-\ldots-p_{n-1}-p_{n}$ and assuming $\mathrm{G}_{\mathrm{m}}(\mathrm{s})=\mathrm{G}(\mathrm{s})$, the elements of $\mathrm{Ko}(\mathrm{s})$ would be given by (22) and (23). The elements in (22) correspond to the zero elements of Qo(s). If $t_{i}(\mathrm{~s})$ has time delay, the Ko(s) elements defined by (23) are irrational transfer functions more difficult to implement. Therefore, some kind of approximation may be required to use this scheme.

$$
\begin{aligned}
& k o_{i j}(s)=g_{i j}(s) \quad \forall j ; \quad i=p_{j} \\
& k o_{i j}(s)=g_{i j}(s)\left(1-\frac{1}{t_{i}(s)}\right) \quad \forall i, j ; / i \neq p_{j}
\end{aligned}
$$


The same result isachieved from the methodology of centralized inverted decoupling control [28] if the desired diagonal open-loop process matrix L(s) is specified in terms of diagonal closed-loop matrix T(s), as in (24).

$$
L(s)=\left(T^{-1}(s)-I\right)^{-1}
$$

According to the centralized inverted decoupling control, expression (25) is obtained. Defining the elements of $\mathrm{Kd}(\mathrm{s})$ as the elements of $\mathrm{Qd}(\mathrm{s})$ in (5), $\mathrm{Ko}(\mathrm{s})$ is given by (26). Calculating its elements for a configuration $p_{1}-p_{2}-\ldots-p_{i}-\ldots-p_{n-1}-p_{n}$, the same expressions (22) and (23) arise.

$$
\begin{aligned}
& K d^{-1}(s)-K o(s)=\left(T^{-1}(s)-I\right) \cdot G(s)=T^{-1}(s) \cdot G(s)-G(s) \\
& K o(s)=G(s)-T^{-1}(s) \cdot G(s)+K d^{-1}(s)
\end{aligned}
$$

In the proposed IMC approach, the tuningprocedure is more straightforward and intuitive than that ofa centralized controller. In addition, simple controller elements are achieved. From a practical point of view, the centralized approach has fewer elements to be implemented; however, the expressions of the Ko(s) controller elements in (23) may need to be approximated into rational transfer functions before implementation.

\subsection{Additional filter}

According to (4), the disturbance rejection performance is governed by the open-loop dynamics of the process $\mathrm{G}(\mathrm{s})$. In order to improve the disturbance rejection response of the closed-loop system, a stable diagonal filter $\mathrm{F}(\mathrm{s})$ is proposed as is shown in the scheme of Figure 5. A similar filter is proposed in the multivariable filtered Smith predictor in [9]. Then, the following closed-loop transfer matrixes $\mathrm{T}(\mathrm{s})$ and $\mathrm{H}(\mathrm{s})$ are obtained (where Laplace variable $s$ has been omitted):

$$
\begin{aligned}
& T=G \cdot Q \cdot\left[I+F\left(G-G_{m}\right) \cdot Q\right]^{-1} \\
& H=G-G \cdot[I+Q \cdot F(G-G m)]^{-1} \cdot Q \cdot F \cdot G
\end{aligned}
$$

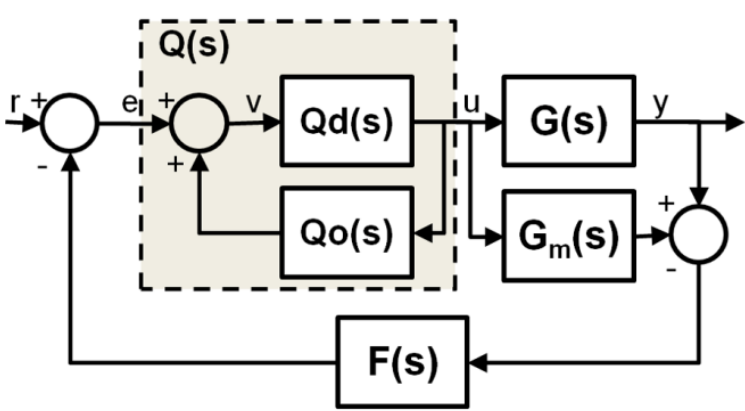

Figure 5. Inverted decoupling IMC scheme with filter. 
For the nominal case $\left(\mathrm{G}(\mathrm{s})=\mathrm{G}_{\mathrm{m}}(\mathrm{s})\right)$, the reference tracking response remains the same $\mathrm{T}(\mathrm{s})=\mathrm{G}(\mathrm{s}) \cdot \mathrm{Q}(\mathrm{s})$, independent of $\mathrm{F}(\mathrm{s})$. Nevertheless, the load disturbance response is modified by the filter as follows:

$$
H=(I-G \cdot Q \cdot F) \cdot G=(I-T \cdot F) \cdot G
$$

Since $\mathrm{T}(\mathrm{s})$ and $\mathrm{F}(\mathrm{s})$ are diagonal matrixes, the matrix [I-T(s)· $\mathrm{F}(\mathrm{s})]$ is diagonal as well. In order to cancel the undesired poles of each row $i$ of $G(s)$, the filter element $f_{i}(\mathrm{~s})$ must be designed in such a way that these slow poles appear as zeros in $\left(1-t_{i}(\mathrm{~s}) \cdot f_{i}(\mathrm{~s})\right)$. This is satisfied if the following condition is fulfilled:

$$
\left.\frac{d^{r}}{d s^{r}}\left(1-t_{i}(s) \cdot f_{i}(s)\right)\right|_{s=z_{k}}=0 \quad r=0,1, \ldots, m_{k}-1 ; k=1, \ldots, p
$$

where $z_{k}$ is an undesired pole, $m_{k}$ is its maximum multiplicity in the row $i$ of $G(\mathrm{~s})$, and $p$ is the total number of undesired poles in the row $i$. In general, the filter element $f_{i}(\mathrm{~s})$ is defined as follows:

$$
f_{i}(s)=\frac{N_{\alpha}(s) \cdot\left(\lambda_{i} s+1\right)^{r}}{\left(\beta_{i} s+1\right)^{\eta}}
$$

where the term $\left(\lambda_{\mathrm{i}} \mathrm{s}+1\right)^{\mathrm{r}}$ cancels in $h_{i}(\mathrm{~s})$ the specified closed-looppoles for reference tracking. The pole in $s=-1 / \beta_{i}$ is used to define the desired time constant of the disturbance rejection response and its degree must be chosen to obtain a proper filter element. Note also that, according to (29), the stationary gain of $f_{i}(\mathrm{~s})$ must be equal to the unity in order to obtain zero steady state error for step disturbance rejection. Then, $\mathrm{N}_{\alpha}(\mathrm{s})=\left[\alpha_{q} \mathrm{~s}^{\mathrm{q}}+\ldots+\alpha_{1} \mathrm{~s}+1\right]$ is a polynomial of the proper degree $q$ and coefficients $\alpha_{k}$ which must be calculated in such a way that $\left[1-t_{i}(\mathrm{~s}) \cdot f_{i}(\mathrm{~s})\right]$ to include as zeros the undesired poles of the original disturbance rejection response.

Therefore, this allows improving the load disturbance response without modifying the nominal tracking response. The filter can be designed easily after determining these desired tracking requirements. This is another advantage of the proposed inverted decoupling IMC over the centralized inverted decoupling control in [28] based on aclassical feedback scheme, where no load disturbance specifications were included in the methodology.

For FOPTD systems with an only undesired pole at $s=-z_{1}$ in $h_{i}(\mathrm{~s})$, the filter is usually defined by (32) and $\alpha_{1}$ is calculated according to (33).

$$
f_{i}(s)=\frac{\left(\alpha_{1} s+1\right)\left(\lambda_{i} s+1\right)}{\left(\beta_{i} s+1\right)^{2}}
$$


$\alpha_{1}=\left[1-\left(1-\beta_{i} \cdot z_{1}\right)^{2} \cdot e^{-\theta_{i} z_{1}}\right] / z_{1}$

As it is shown in $[9,30]$, the filter can also be used to improve the robustness of the system. However, it is shown that there is a trade-off between robustness and disturbance rejection performance. When $\beta_{i}$ decreases, the disturbance rejection becomes faster and the robustness deteriorates, and vice versa. This compromise must be studied for each case. In addition, the filter element $f_{i}(\mathrm{~s})$ can be specified with more poles than zeros to provide low pass behaviour. This reduces the high frequency noise and can increase the robustness.

\subsection{Stability and robustness}

The first condition for stability that the final controller must fulfill is that all of its elements must not have RHP poles. Once the stability of the controller is assured, internal stability of the closed-loop system can be verified if and only if all elements in matrix (34) have all their poles in the left-half plane with no RHP pole-zero cancellation in $\mathrm{G}(\mathrm{s}) \cdot \mathrm{K}(\mathrm{s})[31]$.

$$
\left[\begin{array}{cc}
(I+K(s) \cdot G(s))^{-1} & -K(s) \cdot S(s) \\
S(s) G(s) & S(s)
\end{array}\right]
$$

In this case, $\mathrm{S}(\mathrm{s})$ is the sensitivity transfer function matrix written as $[\mathrm{I}+\mathrm{G}(\mathrm{s}) \cdot \mathrm{K}(\mathrm{s})]^{-1}$. $\mathrm{K}(\mathrm{s})$ can represent the equivalent conventional centralized feedback controller. The proposed method assures this condition if the closed-loop transfer functions are defined according to the conditions in section 2.2 and the process $\mathrm{G}(\mathrm{s})$ has no unstable poles.

To evaluate the robustness of the controller, a $\mu$-analysis can be performed for different types of uncertainties. For instance, multiplicative input uncertainty is illustrated in Figure 6 , where $\Delta_{\mathrm{I}}(\mathrm{s})$ is the disturbance and $\mathrm{W}_{\mathrm{I}}(\mathrm{s})$ and $\mathrm{W}_{\mathrm{P}}(\mathrm{s})$ are the diagonal weights for uncertainty and performance, respectively.

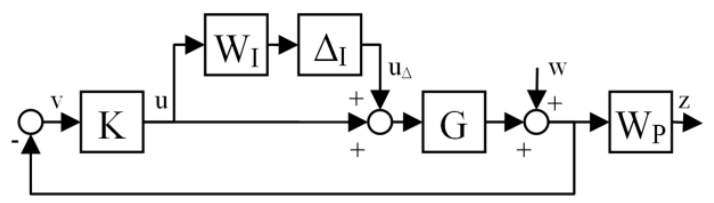

Figure 6.System with multiplicative input uncertainty and performance measured at the output.

To achieve robust stability the necessary and sufficient condition in a classical feedback system [31] is

$$
\mu_{\mathrm{RS}}=\mu\left[-\mathrm{W}_{\mathrm{I}}(\mathrm{s}) \mathrm{T}_{\mathrm{I}}(\mathrm{s})\right]<1 \quad \forall \omega
$$

where $\mu$ is the structured singular value (SSV) and $\mathrm{T}_{\mathrm{I}}(\mathrm{s})=\mathrm{K}(\mathrm{s}) \mathrm{G}(\mathrm{s})(\mathrm{I}+\mathrm{K}(\mathrm{s}) \mathrm{G}(\mathrm{s}))^{-1}$ is the input complementary sensitivity function. To evaluate if the closed loop system will 
respect the desired performance even in presence of diagonal multiplicative input uncertainty, the necessary and sufficient condition [31] is

$$
\mu_{\mathrm{RP}}=\mu\left[\begin{array}{cc}
-\mathrm{W}_{\mathrm{I}}(\mathrm{s}) \mathrm{T}_{\mathrm{I}}(\mathrm{s}) & -\mathrm{W}_{\mathrm{I}}(\mathrm{s}) \mathrm{K}(\mathrm{s}) \mathrm{S}(\mathrm{s}) \\
\mathrm{W}_{\mathrm{P}}(\mathrm{s}) \mathrm{S}(\mathrm{s}) \mathrm{G}(\mathrm{s}) & \mathrm{W}_{\mathrm{P}}(\mathrm{s}) \mathrm{S}(\mathrm{s})
\end{array}\right]<1 \quad \forall \omega
$$

To carry out this analysis, it is necessary to calculate the equivalent conventional centralized feedback controller K(s) according to the structure of Figure 6, and to define the weights. It is important to note that this analysis is performed after the nominal design. As mentioned previously, if the proposed method is used with the filter F(s), it can be tuned in order to improve the robustness; however, the load disturbance response will deteriorate in this case.

\section{Examples}

In this section, three simulation examples are presented to demonstrate the effectiveness of the proposed methodology. The multivariable processes are $2 \times 2$ or $3 \times 3$ stable systems in which the proposed method is applied.

\subsection{Example 1: Heavy oil fractionator}

The transfer function matrix of this $2 \times 2$ process with important time delays is given by (37) in [32]. The time constant and delays are expressed in minutes. It does not have multivariable RHP zeros.

$$
G_{H}(s)=\left(\begin{array}{cc}
\frac{4.05 e^{-27 s}}{27 s+1} & \frac{1.77 e^{-28}}{60 s+1} \\
\frac{5.39 e^{-18 s}}{50 s+1} & \frac{5.72 e^{-14 s}}{60 s+1}
\end{array}\right)
$$

According to the conditions of section 2.2, configuration 1-2 must be chosen for realizability without adding extra dynamics. Since all process elements are FOPTD systems, the desired closed-loop transfer functions $t_{i}$ (s) can be specified as in (16). The time delays must be fixed to 27 in $t_{1}(\mathrm{~s})$ and 14 in $t_{2}(\mathrm{~s})$ in order to meet the realizability condition (11). In [32], a $\mathrm{H}_{2}$ decoupling IMC is developed to control this process using the scheme of Figure 1; the desired closed-loop time constant for reference tracking are specified as 19 and 26 min in loop 1 and 2, respectively. Therefore, the same time constants $\lambda_{1}$ and $\lambda_{2}$ are used for the proposed control in order to carry out a fair comparison between these methodologies. The desired closed-loop transfer functions are defined by

$$
t_{1}(s)=\frac{e^{-27 s}}{19 s+1} \quad t_{2}(s)=\frac{e^{-14 s}}{26 s+1}
$$


Then, the IMC matrixes Qd(s) and Qo(s) are obtained from (7). The resultant controllers are as follows:

$$
\begin{gathered}
\operatorname{Qd}(s)=\left(\begin{array}{cc}
\frac{0.2469 \cdot(27 s+1)}{19 s+1} & 0 \\
0 & \frac{0.1748 \cdot(60 s+1)}{26 s+1}
\end{array}\right) \\
Q o(s)=\left(\begin{array}{cc}
0 & \frac{-1.77 \cdot(19 s+1)}{60 s+1} e^{-s} \\
\frac{-5.39 \cdot(26 s+1)}{50 s+1} e^{-4 s} & 0
\end{array}\right)
\end{gathered}
$$

Using the scheme in Figure 3, this is the proposed inverted decoupling IMC (IDIMC) with $G_{m}(s)$ equal to (37). Figure 7 shows the closed-loop system response in comparison with that of the mentioned $\mathrm{H}_{2}$ decoupling IMC (H2-IMC), which is given by (41).

$$
Q(s)=\left(1193.2 s^{2}+67.4 s+1\right) \cdot\left(\begin{array}{cc}
\frac{0.4198}{(60 s+1)(19 s+1)} & \frac{-0.1299 \cdot e^{-s}}{(60 s+1)(26 s+1)} \\
\frac{-0.3956 \cdot e^{-4 s}}{(50 s+1)(19 s+1)} & \frac{0.2972}{(27 s+1)(26 s+1)}
\end{array}\right)
$$

There is a unit step change in the first reference at $t=0 \mathrm{~min}$, and at $t=200 \mathrm{~min}$, in the second one. At $t=400 \mathrm{~min}$, there is a 0.2 step in both process inputs as load disturbances. The IAE indices are collected in Table 1. The total variation (TV) is also shown as a measure of the control effort. Both controllers achieve almost the same responses, which seem to be overlapped. This is logical because they have been designed for the same performance specifications. Nevertheless, controller elements in (41) are more complicated than those of the proposed method and in addition, they were obtained after some approximations. The proposed design has been carried out in a simpler and easier way without any reduction. 

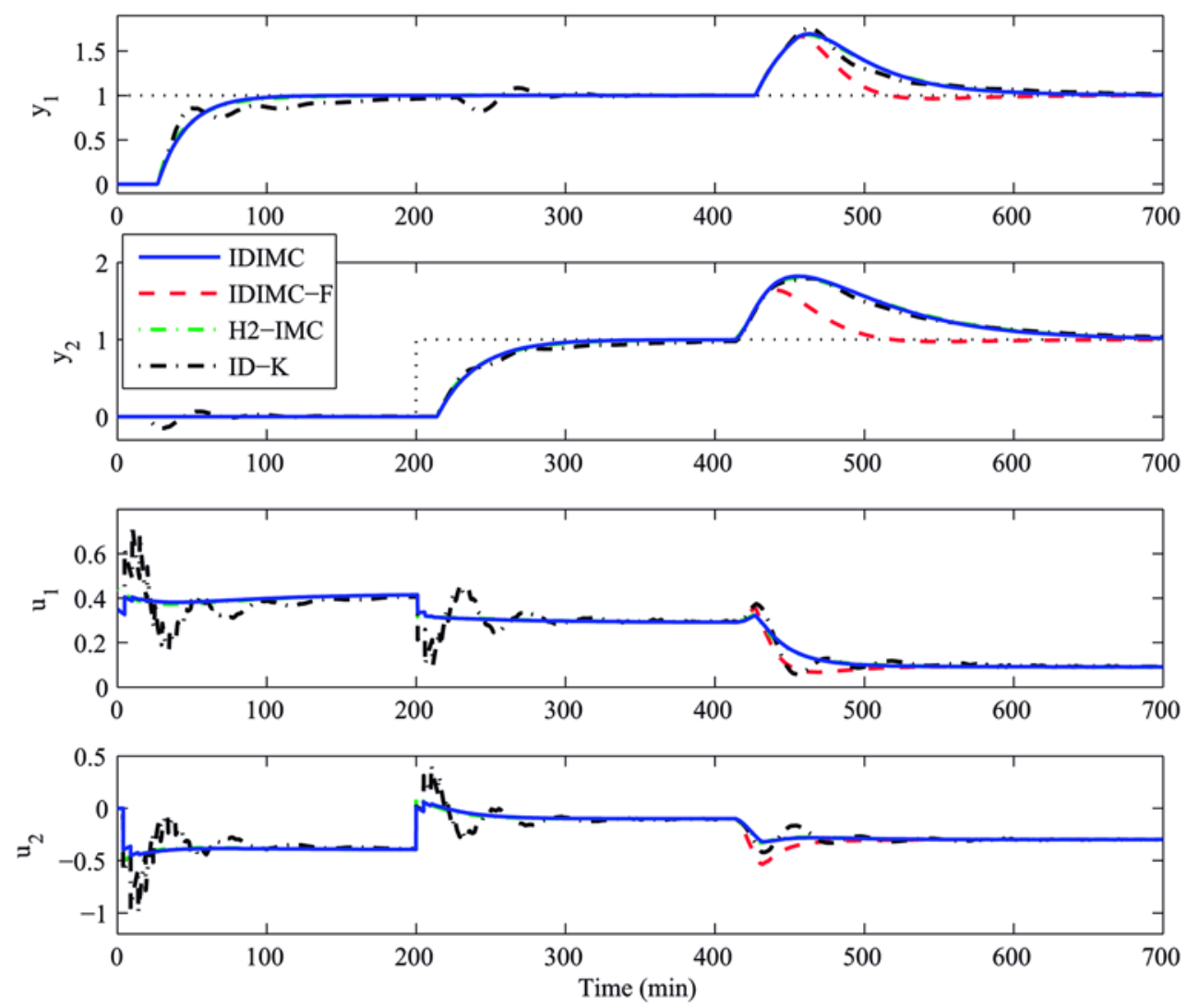

Figure 7. Outputs and control signals of the step response in example 1.

Table 1. IAE values and TV indices for each method in example 1.

\begin{tabular}{lcccccccc}
\hline & \multicolumn{2}{c}{ IDIMC } & \multicolumn{2}{c}{ IDIMC-F } & \multicolumn{2}{c}{ H2-IMC } & \multicolumn{2}{c}{ ID-K } \\
\cline { 2 - 8 } & loop 1 & loop 2 & loop 1 & loop 2 & loop 1 & loop 2 & loop1 & loop 2 \\
\hline IAE & 99.4 & 127.8 & 77.8 & 73.1 & 100.2 & 128.9 & 113.7 & 136.6 \\
TV & 0.6 & 1.18 & 0.72 & 1.59 & 0.52 & 1 & 6.85 & 13.5 \\
$\boldsymbol{\mu}_{\text {RS }}$ & 0.21 & 0.37 & 0.21 & 0.59 \\
$\boldsymbol{\mu}_{\text {RP }}$ & 0.94 & 1.27 & 0.92 & 1.31 \\
\hline
\end{tabular}

With the proposed method, the transfer matrix $\mathrm{H}(\mathrm{s})$ from the load disturbances to the outputs has a slow pole at $s=-1 / 60$ at the first output, and two slow poles at $s=-1 / 50$ and $s=-1 / 60$ in the second one. Thus, the disturbance rejection can be improved with a diagonal filter $\mathrm{F}(\mathrm{s})$, as in the scheme of Figure 5 . This filter is designed to remove these slow poles from $\mathrm{H}(\mathrm{s})$ and define the desired disturbance rejection time constants. Time constants have been chosen to be similar to those of reference tracking. The filter element $f_{1}$ (s) is obtained according to (32) and (33) because there is only one slow pole associated to the output 1 . The filter element $f_{2}(\mathrm{~s})$ needs two degrees of freedom, and it is calculated by fulfilling condition (30) for the poles $s=-1 / 50$ and $s=-1 / 60$, simultaneously. The resultant diagonal filter is as follows: 


$$
F(s)=\left(\begin{array}{cc}
\frac{42.12 s+1}{(19 s+1)} & 0 \\
0 & \frac{1660.052 s^{2}+79.022 s+1}{(26 s+1)^{2}}
\end{array}\right)
$$

In Figure 7, the step response of the proposed method with filter (IDIMC-F) is also shown and the IAE values are listed in Table 1. The disturbance rejection response is improved without affecting the nominal reference tracking response. In contrast, the TV indices increase slightly.

Figure 7 also shows the step response of the initial proposed control (IDIMC) when it is implemented using the equivalent centralized controller (ID-K), as in Figure 4. With this scheme, $\mathrm{Kd}(\mathrm{s})$ matrix is equal to $\mathrm{Qd}(\mathrm{s})$, the diagonal elements of $\mathrm{Ko}(\mathrm{s})$ are calculated according to (22), and the off-diagonal ones according to (23). However, these off-diagonal elements result in irrational transfer functions because of time delays, and they are approximated. The achieved Ko(s) matrix is given by (43). With this controller, the step response of second loop is quite similar to that obtained with the first proposed method, although it shows some interactions. The first loop response shows a bit sluggish behavior and the control signals are rather oscillatory. The corresponding IAE values are collected in Table 1, where they are the greatest ones. This can be expected from the approximation of the off-diagonal Ko(s) elements. In addition, the TV values are also significantly higher.

$$
K o(s)=\left(\begin{array}{cc}
\frac{4.05 e^{-27 s}}{27 s+1} & \frac{-81.42 s}{60 s+1} e^{-s} \\
\frac{-215.6 s}{50 s+1} e^{-4 s} & \frac{5.72 e^{-14 s}}{60 s+1}
\end{array}\right)
$$

In order to evaluate the robustness, a $\mu$-analysis can be performed according to section 2.5. In this example, the chosen weights are given by (44). The weight $w_{I}(\mathrm{~s})$ can be loosely interpreted as the process inputs increase by up to $100 \%$ uncertainty at high frequencies and by almost $20 \%$ uncertainty in the low frequency range. The performance weight $w_{P}(\mathrm{~s})$ specifies integral action, a maximum peak for the singular value of the sensitivity transfer matrix of $M_{s}=2.2$ and a bandwidth of about $0.001 \mathrm{rad} / \mathrm{s}$.

$$
\begin{aligned}
& \mathrm{W}_{\mathrm{I}}(\mathrm{s})=\mathrm{W}_{\mathrm{I}}(\mathrm{s}) \cdot \mathrm{I}=\frac{(\mathrm{s}+0.2)}{\mathrm{s}+1} \cdot \mathrm{I} \\
& \mathrm{W}_{\mathrm{P}}(\mathrm{s})=\mathrm{W}_{\mathrm{P}}(\mathrm{s}) \cdot \mathrm{I}=\frac{(\mathrm{s} / 2.2+0.001)}{\mathrm{s}} \cdot \mathrm{I}
\end{aligned}
$$

The SSV for robust stability and robust performance for the different controllers are shown in Figure 8. The proposed IDIMC control obtains similar robust stability (RS) and robust performance (RP) than those of the H2IMC control. They fulfill conditions (35) and (36) for all frequencies, indicating that the systems will remain stablein spite of an uncertainty of $20 \%$ on each process input. The peak values are shown in Table 1 . For 
the proposed IDIMC-F control, the RS and RP will deteriorate at middle frequencies, where the peaks appear, because there is a trade-off between robustness and disturbance rejection, as it was mentioned at the end of section 2 [9]. These peak values are also collected in Table 1. The approximated ID-K control shows the worst RS and RP because of the approximations performed in its design.
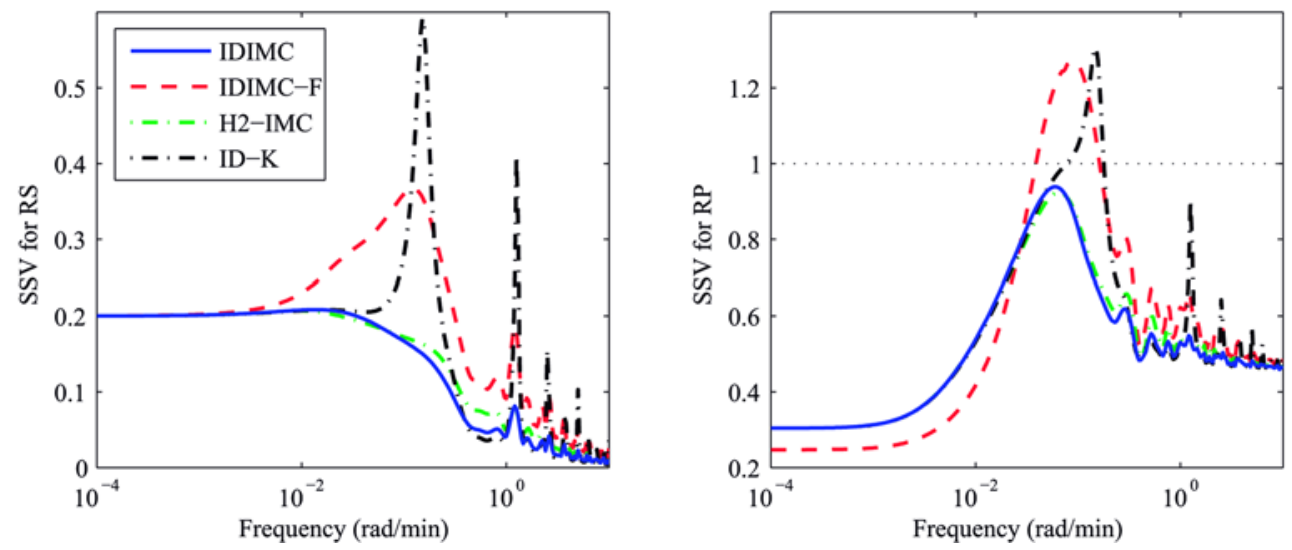

Figure 8.SSV for robust stability and robust performance in example 1.

\subsection{Example 2: Jerome and Ray process}

The transfer matrix of this $2 \times 2$ process is given by (45). It has important time delays and multivariable RHP zeros at $s=1$. Nevertheless, the proposed methodology can be applied because this zero is associated to both outputs explicitly.

$G_{J}(s)=\left(\begin{array}{cc}\frac{(-s+1) e^{-2 s}}{s^{2}+1.5 s+1} & \frac{0.5(-s+1) e^{-4 s}}{(2 s+1)(3 s+1)} \\ \frac{0.33(-s+1) e^{-6 s}}{(4 s+1)(5 s+1)} & \frac{(-s+1) e^{-3 s}}{4 s^{2}+6 s+1}\end{array}\right)$

According to section 2.2, configuration 1-2 must be selected for realizability without adding extra dynamics. The desired closed-loop transfer functions $t_{i}(\mathrm{~s})$ are specified under (14). Due to conditions (12) and (13), their relative degreeand the multiplicity of the RHP zero with its mirrored pole must be one. According to (11), the minimum time delays are $2 \mathrm{~s}$ in loop 1 , and $3 \mathrm{~s}$ in the loop 2 . A desired closed-loop time constant of $1 \mathrm{~s}$ is specified for both loops. Then, the desired $t_{i}(\mathrm{~s})$ are defined as follows:

$t_{1}(s)=\frac{(-s+1) e^{-2 s}}{(s+1)^{2}} \quad t_{2}(s)=\frac{(-s+1) e^{-3 s}}{(s+1)^{2}}$

By means of (7), the IMC matrixes Qd(s) and Qo(s) of the proposed method are calculated obtaining the matrixes given by (47) and (48). 


$$
\begin{aligned}
& \operatorname{Qd}(s)=\left(\begin{array}{cc}
\frac{s^{2}+1.5 s+1}{(s+1)^{2}} & 0 \\
0 & \frac{4 s^{2}+6 s+1}{(s+1)^{2}}
\end{array}\right) \\
& \operatorname{Qo}(s)=\left(\begin{array}{cc}
0 & \frac{-0.5 \cdot(s+1)^{2}}{(2 s+1)(3 s+1)} e^{-2 s} \\
\frac{-0.33 \cdot(s+1)^{2}}{(4 s+1)(5 s+1)} e^{-3 s} & 0
\end{array}\right)
\end{aligned}
$$

In order to improve the disturbance rejection response of the second output, a filter $f_{2}(\mathrm{~s})$ is designed in order to cancel the poles $s=-1 / 5$ and $s=-1 / 4$ in the second row of $\mathrm{H}(\mathrm{s})$. A desired time constant $\beta_{2}$ equal to $2 \mathrm{~s}$ is specified for disturbance rejection. Then, the filter element is calculated as is explained in section 2.4. The resultant diagonal filter $\mathrm{F}(\mathrm{s})$ is as follows:

$$
F(s)=\left(\begin{array}{cc}
1 & 0 \\
0 & \frac{\left(17.4963 s^{2}+8.1041 s+1\right)(s+1)}{(2 s+1)^{3}}
\end{array}\right)
$$

The closed-loop system responses of the proposed method without filter (IDIMC) and with it (IDIMC-F) are shown in Figure 9. There are unit step changes at $t=1 \mathrm{~s}$ in the first reference, at $t=50 \mathrm{~s}$ in the second one, and a $0.5 \mathrm{step}$ in both process inputs as load disturbances at $t=100 \mathrm{~s}$. For comparison, the analytical two degrees of freedom decoupling control of Liu in [23] is also presented, which had already demonstrated its superiority over the multivariable Smith predictor of Wang in [25] and the decoupling multivariable control with two degrees freedom in [24]. The IAE values are listed in Table 2. 

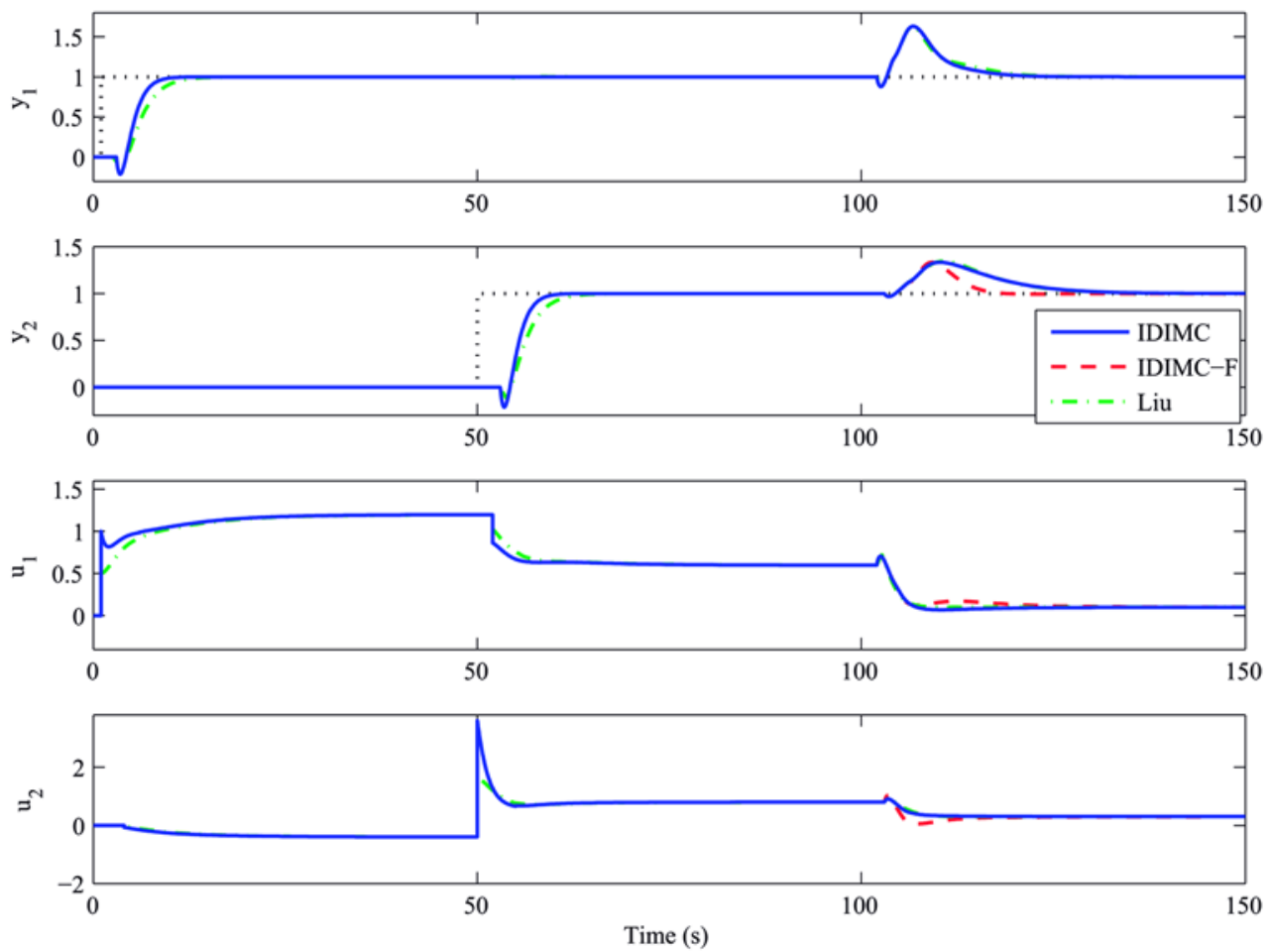

Figure 9. Outputs and control signals of the step response in example 2.

Table 2. IAE values for each method in example 2.

\begin{tabular}{lcccccc}
\hline & \multicolumn{2}{c}{ IDIMC } & \multicolumn{2}{c}{ IDIMC-F } & \multicolumn{2}{c}{ Liu } \\
\cline { 2 - 6 } & loop 1 & loop 2 & loop 1 & loop 2 & loop 1 & loop 2 \\
IAE & 8.95 & 10.05 & 8.95 & 8.08 & 10.25 & 11.1 \\
TV & 2 & 4.3 & 2.1 & 5.1 & 2 & 2.1 \\
$\boldsymbol{\mu}_{\text {RS }}$ & 0.56 & 1.17 & 0.56 \\
$\boldsymbol{\mu}_{\text {RP }}$ & 1.31 & 2.5 & 1.31 \\
\hline
\end{tabular}

The proposed designs achieve similar performance than that of Liu, with a decoupling response, bit smaller settling times and smaller IAE values. In addition, the controller elements are much simpler than those of Liu's controller, and they are calculated easier. With filter in (49), the proposed design maintains the same reference tracking response and improves the rejection of the load disturbances in the second output.

Using the weights in (50), a $\mu$-analysis similar to the previous example is carried out to investigate the robustness. It shows that the proposed IDIMC control and the Liu's controller have similar RS and RP. They remain stable for all frequencies; however, the $\mathrm{RP}$ will deteriorate at middle frequencies around $1 \mathrm{rad} / \mathrm{s}$ where the RP values are greater than one and condition (36) is not fulfilled. The peak values are listed in Table 2. On the other hand, the proposed IDIMC-F control shows the worst RS and RP at frequencies around $1 \mathrm{rad} / \mathrm{s}$. It does not fulfill RS condition (35) at these frequencies, and it has an important peak value for RP. This control improves the disturbance rejection at the expense of decreasing the robustness, as it was commented. 


$$
\begin{aligned}
& W_{I}(s)=W_{I}(s) \cdot I=\frac{(s+0.1)}{s+1} \cdot I \\
& W_{P}(s)=W_{P}(s) \cdot I=\frac{(s / 2.5+0.001)}{s} \cdot I
\end{aligned}
$$

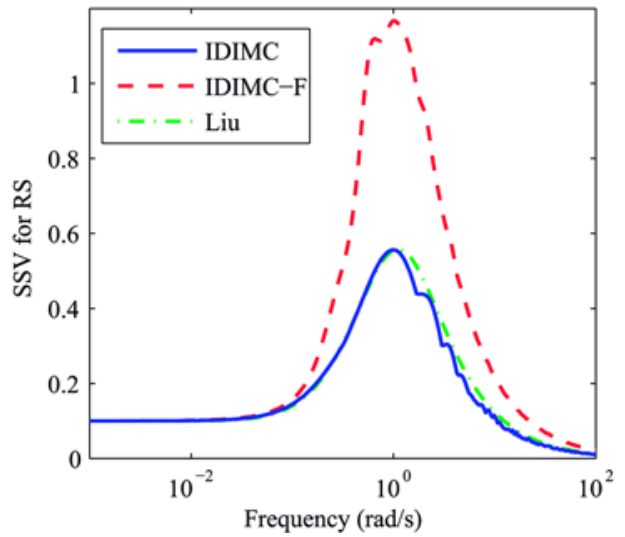

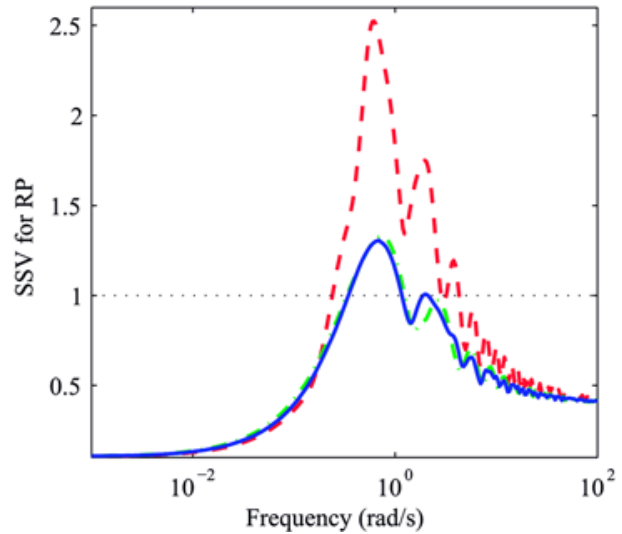

Figure10.SSV for robust stability and robust performance in example 2.

\subsection{Example 3: 3×3 Tyreus distillation column}

The transfer matrix of thisprocess is given by (51) in [28, 33]. Due to time delays, there are no realizable configurations according to section 2.2. Therefore, it is necessary to include an additional block N(s) with delays. To obtain realizability by adding the minimum quantity of delays, the only choice is configuration 1-2-3 with $n_{11}(\mathrm{~s})=e^{-0.09 \mathrm{~s}}$, $n_{22}(\mathrm{~s})=1$ and $n_{33}(\mathrm{~s})=e^{-0.26 \mathrm{~s}}$. Then, the proposed methodology is applied to the new augmented process $\mathrm{G}(\mathrm{s}) \cdot \mathrm{N}(\mathrm{s})$ given by (52).

$$
\mathrm{G}_{\mathrm{T}}(\mathrm{s})=\left(\begin{array}{ccc}
\frac{1.986 e^{-0.71 s}}{66.7 s+1} & \frac{-5.24 e^{-60 s}}{400 s+1} & \frac{-5.984 e^{-2.24 s}}{14.29 s+1} \\
\frac{-0.0204 e^{-0.59 s}}{(7.14 s+1)^{2}} & \frac{0.33 e^{-0.68 s}}{(2.38 s+1)^{2}} & \frac{-2.38 e^{-0.42 s}}{(1.43 s+1)^{2}} \\
\frac{-0.374 e^{-7.75 s}}{22.22 s+1} & \frac{11.3 e^{-3.79 s}}{(21.74 s+1)^{2}} & \frac{9.811 e^{-1.59 s}}{11.36 s+1}
\end{array}\right)
$$

The process elements of the first and third rows are FOPTD systems, and consequently, the corresponding closed-loop transfer functions $t_{1}(\mathrm{~s})$ and $t_{3}(\mathrm{~s})$ can be specified according to (16). On the other hand, the system elements of the second row have relative degree equal to two, and therefore, the desired pole at $s=-1 / \lambda_{2}$ must be defined 
with multiplicity equal to two. Closed-loop time constants of 15, 12 and 18 min are specified in each loop, respectively. The desired closed-loop transfer functions are as follows:

$$
t_{1}(s)=\frac{e^{-0.8 s}}{(15 s+1)} \quad t_{2}(s)=\frac{e^{-0.68 s}}{(12 s+1)^{2}} \quad t_{3}(s)=\frac{e^{-1.85 s}}{(18 s+1)}
$$

Assuming $G_{m}(s)=G_{T}^{N}(s)$ and after selecting the configuration 1-2-3, the controller elements of Qd(s) and Qo(s) are obtained according to (9) and (10) without approximations, as follows:

$$
\begin{aligned}
& Q d(s)=\left(\begin{array}{ccc}
\frac{(66.7 s+1)}{1.986(15 s+1)} & 0 & 0 \\
0 & \frac{(2.38 s+1)^{2}}{0.33(12 s+1)^{2}} & 0 \\
0 & 0 & \frac{(11.36 s+1)}{9.811(18 s+1)}
\end{array}\right) \quad(54) \\
& Q O(s)=\left(\begin{array}{ccc}
\frac{5.24(15 s+1) e^{-5.2 s}}{(400 s+1)} & \frac{5.984(15 s+1) e^{-1.7 s}}{(14.29 s+1)} \\
\frac{0.0204(12 s+1)^{2}}{(7.14 s+1)^{2}} & \frac{-11.3(18 s+1) e^{-1.94 s}}{(21.74 s+1)^{2}} & \frac{2.38(12 s+1)^{2}}{(1.43 s+1)^{2}} \\
\frac{0.374(18 s+1) e^{-5.99 s}}{22.22 s+1} & \frac{0}{(2.25}
\end{array}\right)
\end{aligned}
$$

Although the process is a $3 \times 3$ system, the complexity of the elements of (54) and (55) is as simple as that of the elements obtained for $2 \times 2$ processes. The closed-loop system response is shown in Figure 11. There are unit step changes at $t=1 \mathrm{~min}$ in the first reference, at $t=333 \mathrm{~min}$ in the second one, and at $t=666 \mathrm{~min}$ in the third one.For comparison, other control methodologies are also shown: the pure centralized control of Wang in [34] and the analytical two degrees of freedom decoupling control of Liu in [23].

The proposed design achieves perfect decoupling performance without overshoot or inverse response in the outputs. It achieves similar IAE values than those of Liu's controller with a similar response. The Wang's controller obtains the worst IAE values, although it has the smaller TV values. These values are listed in Table 3. However, it is important to note that the complexity of the controller elements of Wang or Liu is much greater than that of the proposed control in (54) and (55). They have a high order about fourth, five or six. In addition, these design procedures are more complex than that of the proposed one. 

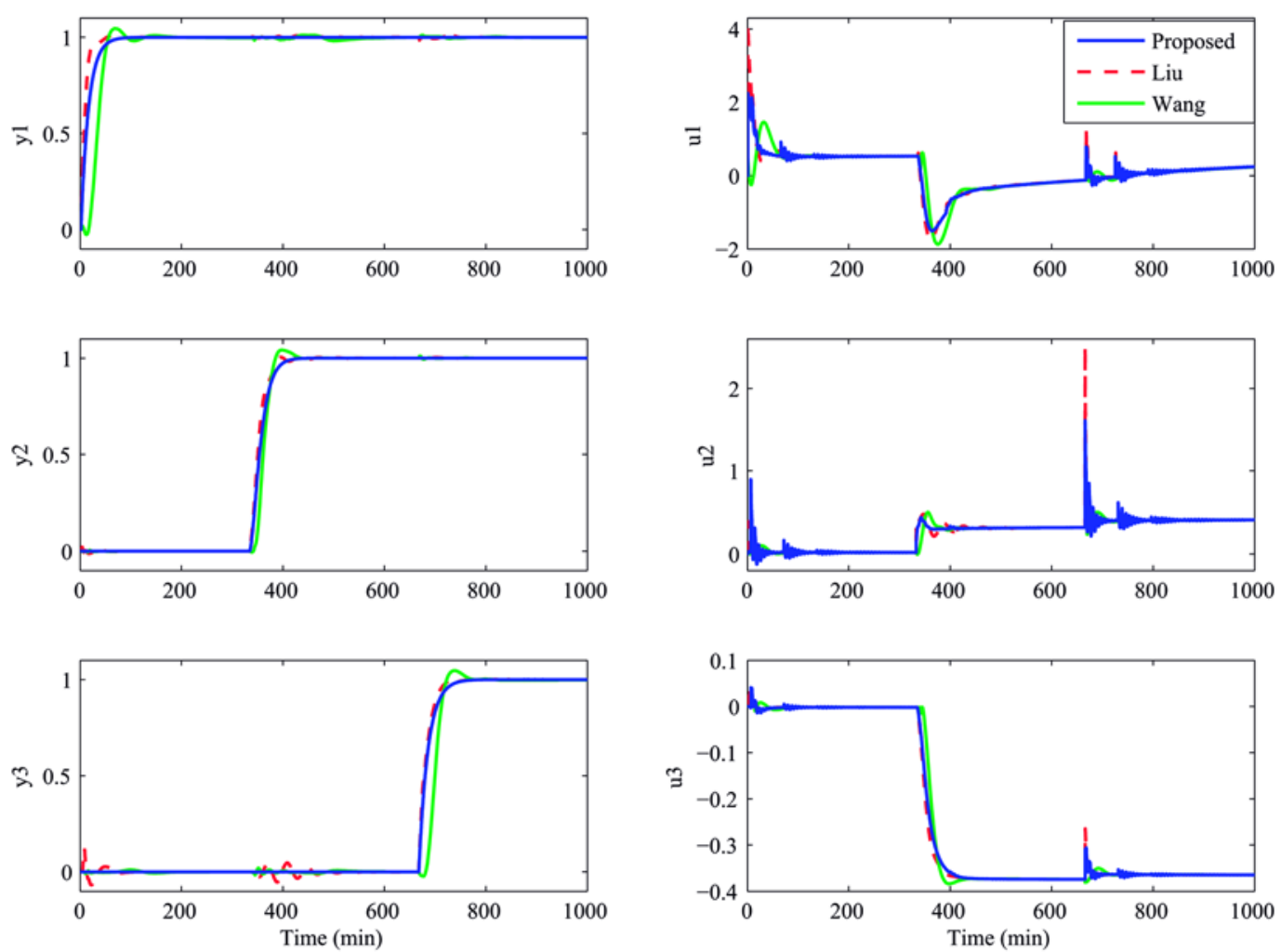

Figure 11. Outputs and control signals of the step response in example 3.

Table 3. IAE values for each method in example 3.

\begin{tabular}{ccccccc}
\hline Method & $\mathbf{I A E}_{\mathbf{1}}$ & $\mathbf{I A E}_{\mathbf{2}}$ & $\mathbf{I A E}_{\mathbf{3}}$ & $\mathbf{T V}_{\mathbf{1}}$ & $\mathbf{T V}_{\mathbf{2}}$ & $\mathbf{T V}_{\mathbf{3}}$ \\
\hline Proposed & 15 & 25 & 20 & 25 & 14 & 1.2 \\
Liu & 10 & 22 & 22 & 290 & 3.6 & 1 \\
Wang & 38.7 & 31 & 38.3 & 9.5 & 1.7 & 0.5 \\
\hline
\end{tabular}

\section{Conclusions}

A new tuning methodology of decoupling IMC formultivariable square and stable processes with multiple time delays has been developed in this work. It is based on the centralized inverted decoupling structure, and from a compact matrix formulation, the generalized expressions for $n \times n$ processes have been obtained. From them, it is shown that the controller elements are very simple. Additionally, it is found that the complexity of these controller elements is independent of the system size.

The realizability conditions for applying the method were provided. In order to improve the disturbance rejection response, a diagonal filter is proposed to be added, and it is demonstrated that it does not affect the nominal reference tracking response. 
The method has been illustrated through three simulation examples,and comparisonswith other works have demonstrated that the proposed methodology achieves similar or better performance. In addition, the obtained controllers are simpler and they have been designed in a more direct way.

\section{Acknowledgements}

This work was supported by the Autonomous Government of Andalusia (Spain) under the Excellence Project P10-TEP-6056; and the Spanish Ministry of Economy and Competitiveness under Grant DPI2012-37580-C02-01.

\section{References}

[1] J.E. Normey-Rico, E.F. Camacho, Control of dead-time processes, Springer-Verlag, 2007.

[2] O.J.M. Smith, Closed control of loops with dead time, Chem. Eng. Prog. 53 (1957) 217-219.

[3] Z.J. Palmor, Time delay compensation: Smith predictor and its modifications, in: The Control Hand Book, CRC Press and IEEE Press, 1996, pp. 224-237.

[4] J.E. Normey-Rico, E.F. Camacho, Predicción para control: Una panorámica del control de procesos con retardo, Rev. Iberoam. Autom. Inform. Ind. 3 (2006) 5-25.

[5] N.F. Jerome, W.H. Ray, High-performance multivariable control strategies for systems having time delays, AIChE J. 32 (1986) 914-931.

[6] Q.G. Wang, Y. Zhang, M.S. Chiu, Decoupling internal model control for multivariable systems with multiple time delays, Chem. Eng. Sci. 57 (2002) 115-124.

[7] A.S. Rao, M. Chindambaram, Smith delay compensator for multivariable non-square systems with multiple time delays, Comput. Chem. Eng. 30 (2006) 1243-1255.

[8] R.S. Sánchez-Peña, Y. Bolea, V. Puig, MIMO Smith predictor: global and structured robust performance analysis, J. Process Control 19 (2009) 163-177.

[9] R.C.C. Flesch, B.C. Torrico, J.E. Normey-Rico, M.U. Cavalcante, Unified approach for minimal output dead time compensation in MIMO processes, J. Process Control 21 (2011) 1080-1091.

[10] Q.B. Jin, F. Hao, Q. Wang, A multivariable IMC-PID method for non-square large time delay systems using NPSO algorithm, J. Process Control 23 (2013) 649-663.

[11] T. Liu, W. Zhang, D. Gu, Analytical design of decoupling internal model control (IMC) scheme for two-input-two-output (TITO) processes with time delays, Ind. Eng. Chem. Res. 45 (2006) 3149-3160.

[12] M. Morari, E. Zafiriou, Robust Process Control, Prentice-Hall, 1989.

[13] Q.G. Wang, Y. Zhang, M.S. Chiu, Non-interacting control design for multivariable industrial processes, J. Process Control 13 (2003) 253-265.

[14] T. Liu, W. Zhang, F. Gao, Analytical decoupling control strategy using a unity feedback control structure for MIMO processes with time delays, J. Process Control 17 (2007) 173-186.

[15] F. Morilla, J. Garrido, F. Vázquez, Control Multivariable por Desacoplo, Rev. Iberoam. Autom. Inform. Ind. 10 (2013) 3-17.

[16] L.B. Xie, L.S. Shiehb, F. Pana, J.S.H. Tsaic, J.I. Canelond, Design of decoupling and tracking controllers for continuous-time transfer function matrices with multiple time delays, J. Process Control 24 (2014) 152-170.

[17] Q. Xiong, W.J. Cai, M.J. He, Equivalent transfer function method for PI/PID controller design of MIMO processes, J. Process Control 17 (2007) 665-673.

[18] V. Vijay-Kumar, V.S.R. Rao, M. Chidambaram, Centralized PI controllers for interacting multivariable processesby synthesis method, ISA Trans. 51 (2012) 400-409.

[19] G. Galdos, A. Karimi, R. Longchamp, $\mathrm{H}_{\infty}$ Controller design for spectral MIMO models by convex optimization, J. Process Control 20 (2010) 1175-1182.

[20] F. Vázquez, F. Morilla, S. Dormido, An iterative method for tuning decentralized PID controllers, in: Proceedings of the 14th IFAC World Congress, Beijing, China, 1999, pp. 491496.

[21] H.P. Huang, J.C. Jeng, C.H. Chiang, W. Pan, A direct method for multi-loop PI/PID controller design, J. Process Control 13 (2003) 769-786. 
[22] M.-J. He, W.-J. Cai, B.-F. Wu, Design of decentralized IMC-PID controller based on dRI analysis, AIChE J. 52 (2006) 3852-3863.

[23] T. Liu, W. Zhang, Analytical two-degrees-of-freedom (2-DOF) decoupling control scheme for multiple-input-multiple-output (MIMO) processes with time delays, Ind. Eng. Chem. Res. 46 (2007) 6546-6557.

[24] H.P. Huang, F.Y. Lin, Decoupling multivariable control with two degrees of freedom, Ind. Eng. Chem. Res. 45 (2006) 3161-3173.

[25] Q.G. Wang, B. Zou, Y. Zhang, Decoupling Smith predictor design for multivariable systems with multiple time delays, Chem. Eng. Res. Des.78 (2000) 152-170.

[26] J. Qibing, Q, Ling, Q. Fei, W. Xuewei, Internal Model Control for Multivariable Coupling System with Time Delays and Optimization Research, in: 2010 International Conference on Measuring Technology and Mechatronics Automation, IEEE, 2010, pp. 505-509.

[27] W. Zhang, L. Chen, L. Ou, Algebraic Solution to $\mathrm{H}_{2}$ Control Problems. II. The Multivariable Decoupling Case, Ind. Eng. Chem. Res. 45 (2006) 7163-7176.

[28] J. Garrido, F. Vázquez, F. Morilla, Centralized Inverted Decoupling Control, Ind. Eng. Chem. Res. 52 (2013) 7854-7866.

[29] J. Garrido, F. Vázquez, F. Morilla, An extended approach of inverted decoupling, J. Process Control21 (2011) 55-68.

[30] J.E. Normey-Rico, E.F. Camacho, Unified approach for robust dead-time comensator design, J. Process Control19 (2009) 38-47.

[31] S. Skogestad, I. Postlethwaite, Multivariable Feedback Control: Analysis and Design, 2 ed., John Wiley \& sons, 2005.

[32] W. Zhang, Quantitative Process Control Theory: CRC Press, 2012.

[33] B.D. Tyreus, Multivariable control system design for an industrial distillation column, Ind. Eng. Chem. Process Des. Dev. 18(1979) 177-182.

[34] Q.G. Wang, Decoupling Control, Lecture Notes in Control and Information Sciences, SpringerVerlag, 2003. 